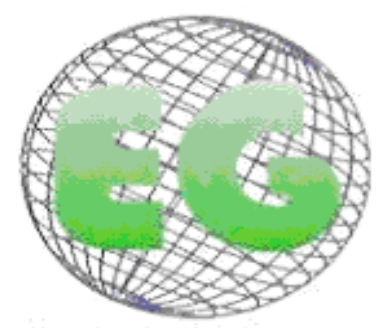

ISSN 1695-6141 N 27
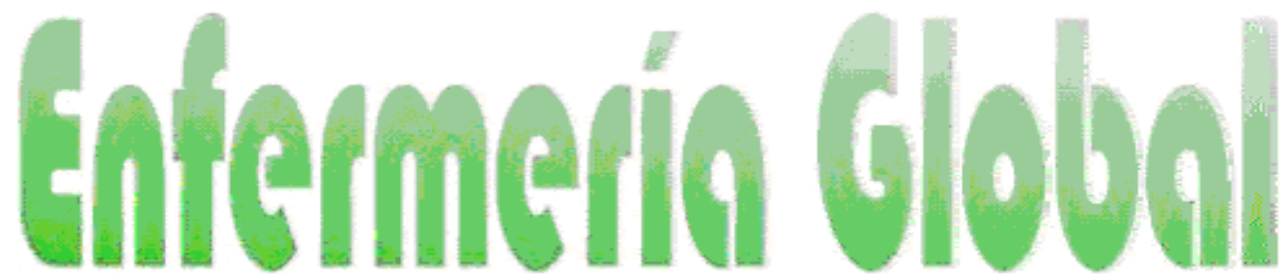

Revista electrónica trimestral de Enfermería

Julio 2012

www.um.eslegloball

\title{
Experiencia de transferencia tecnológica de la Consejería Telefónica para Cuidados Crónicos de Salud, a equipos de Atención Primaria
}

Experience of technology transfer from Telephone Counseling for Chronic Conditions to Primary Care Teams

\section{*Bustamante Troncoso, C., "*Riquelme Hernández, G., " ${ }^{* * *}$ Alcayaga Rojas, C., ****Lange Haensgen, I., ****Urrutia Bunster, M.}

\begin{abstract}
*Enfermera Matrona. Magíster en Enfermería. Profesora Asistente. E-mail: cqbustam@puc.cl ${ }^{* *}$ Matrona. Magíster en Enfermería. Profesora Asistente Adjunto. ${ }^{* * *}$ Enfermera Matrona. Magíster en Psicología de la Salud. Profesora Asistente Adjunto. ${ }^{* * * *}$ Enfermera. Profesora Titular. Escuela de Enfermería. Facultad de Medicina Pontificia Universidad Católica de Chile.
\end{abstract}

Palabras clave: Transferencia tecnológica; enfermedades crónicas; apoyo telefônico; automanejo. Keywords: Technology transfer; chronic diseases; telephone counseling; self-management.

\section{RESUMEN}

El proceso de transferencia tecnológica exige capacitar en conocimientos y habilidades específicas a los futuros usuarios, así como también la realización de adecuaciones administrativas para la incorporación efectiva de la innovación en la estructura del sistema y en la atención profesional.

La Escuela de Enfermería a la que pertenecen los autores, desarrolló un modelo de Apoyo Tecnológico para el Autocuidado en Salud que incluye el uso de consejerías telefónicas para apoyar a personas con enfermedades crónicas en el automanejo y autocuidado de su condición. Se presenta a continuación la experiencia llevada a cabo para transferir esta tecnología a profesionales del Programa de Salud Cardiovascular en Centros de Atención Primaria públicos de una comuna de bajos recursos en Chile, para su incorporación en la atención de pacientes con diabetes tipo 2 adscritos a dicho programa.

Este proceso se realizó en dos etapas: 1. Realización de un taller teórico práctico en apoyo a la toma de decisiones en salud y estrategias motivacionales para el cambio de conducta en salud (talleres y demostraciones) y 2 . Demostración en terreno, y seguimiento con supervisión y reuniones a nivel local.

Los profesionales que participaron del programa de transferencia tecnológica, lograron desarrollar las destrezas esperadas en la herramienta de Consejería Telefónica de Apoyo al Auto-Manejo de Personas con DM2. Finalmente, para incorporar las consejerías telefónicas en la práctica clínica, los centros de salud reestructuraron el sistema de prestación de servicios, integrando así dichas consejerías a su quehacer profesional. 


\section{ABSTRACT}

The technology transfer process requires training future users in specific knowledge and skills, as well as performing administrative adjustments for an effective incorporation of innovation into the structure of the system and into the professional care.

Pontificia Universidad Católica de Chile's School of Nursing developed a model of Technological Support for Health Self-Care, which includes the use of telephone counseling to help people who suffer from chronic diseases manage their condition and look after themselves by teaching them techniques which alleviate their disease. Here, we present an experience that was carried out to transfer this technology to professionals related to the Cardiovascular Health Program in Chilean Public Primary Healthcare Centers located in a low-income district of Chile's capital city, Santiago, so that the technology could be applied to patients with type 2 diabetes who had joined such program.

This process was conducted in two stages: 1. Making of a theoretical-practical workshop to support health decision-making and motivational strategies for health behavior change (workshops and demonstrations); and 2. Field demonstration, and monitoring with supervision and meetings at local level.

The professionals who participated in the technology transfer program succeeded in developing the skills expected in the Telephone Counseling Tool to Support Self-Management of People suffering from DM2. Finally, to enable telephone counseling in clinical practice, healthcare centers restructured their system of service provisions, incorporating such counseling into their professional duties.

\section{INTRODUCCIÓN}

La innovación es un imperativo para la enfermería de hoy. El Consejo Internacional de Enfermeras (CIE) indica que: Las soluciones nuevas ofrecidas por las enfermeras son un elemento vital de las iniciativas encaminadas a abordar los problemas de salud mundiales, actuales y futuros -problemas tales como el envejecimiento de la población, el VIH/SIDA, la tuberculosis, el paludismo, el aumento de las enfermedades no transmisibles, la pobreza, los recursos inadecuados y la escasez de recursos humanos ${ }^{(1)}$.

Sin embargo, de acuerdo a lo que reporta la literatura científica, la puesta en práctica de nuevos conceptos y programas probados demora en llegar a formar parte de la práctica 0 simplemente, no llega a serlo. Muchas veces los profesionales sienten que las intervenciones basadas en la investigación son difíciles de introducir en el ámbito comunitario, y que los fundamentos de la investigación no siempre responden a las necesidades y contexto donde se desempeñan los clínicos ${ }^{(2)}$.

La transferencia tecnológica involucra, además de la nueva tecnología en sí, diversos componentes y formatos cognitivos que son necesarios para lograr que se instale en la práctica. Según Estébanez y Korsunsky, entre estos componentes se encuentran: a) provisión de formación continua y enseñanza profesional para la adquisición de nuevos conocimientos, b) entrenamiento y desarrollo de habilidades en áreas específicas, c) asistencia específica para la resolución de problemas concretos entre investigadores y quienes solicitaron la investigación, d) provisión de conocimiento en áreas interdisciplinarias y áreas de rápido cambio y crecimiento y e) difusión, extensión e intercambio del conocimiento y la información científica básica, tanto a los usuarios e investigadores interesados, como a los profesionales, expertos y público en general ${ }^{(3)}$.

El proceso de transferencia tecnológica considera la capacitación de los futuros usuarios de la tecnología y el acompañamiento durante la puesta en marcha, a fin de ir, en conjunto, realizando las adecuaciones necesarias de la misma tecnología y de la estructura y sistema 
administrativo de la institución, para que ésta sea realmente un aporte y se articule fácilmente con el quehacer profesional.

Por otra parte, en el ámbito del cuidado de personas con enfermedades crónicas (EC), se observa que la atención de pacientes con EC es poco efectiva. Múltiples condiciones influyen en ello, desde la forma de entrega de cuidados de los profesionales de la salud, aún con un fuerte enfoque biomédico, hasta la organización de servicios de salud, caracterizada por prestaciones aisladas y discontinuas. Este enfoque impacta directamente en la calidad de la interacción entre los usuarios y profesionales y específicamente, en los resultados factibles de obtener a partir de ella. Una interacción deteriorada o interferida por aspectos deficientes, puede mermar entre otros aspectos, la adherencia al tratamiento y la satisfacción de los usuarios ${ }^{(2) .}$

La literatura científica indica que, la participación del usuario es la clave para un control exitoso de la enfermedad. En este sentido, el rol de los profesionales es proveer apoyo para los procesos de toma de decisiones en salud de los pacientes ${ }^{(4)}$. Por ello, es fundamental buscar estrategias que favorezcan la adquisición de habilidades en los profesionales de manera que les permitan brindar un apoyo efectivo ${ }^{(5)}$.

La Escuela de Enfermería a la que pertenecen los autores, asumió este desafío y desarrolló un modelo de Apoyo Tecnológico para el Autocuidado en Salud (ATAS) que incluye el uso de consejerías telefónicas para apoyar a personas con EC en el automanejo y autocuidado de su condición. Este modelo, que complementa la atención tradicional otorgada por los centros de salud ambulatorios, se implementó y evaluó con aportes estatales provenientes del Fondo de Fomento para el Desarrollo Científico y Tecnológico (FONDEF). Sus resultados positivos motivaron a las autoridades de la Comuna a incorporar el modelo en la atención a sus usuarios en uno de los centros de salud, que se transformaría en un centro de demostración del uso de dicha tecnología ${ }^{(6-7)}$. Para ello se desarrolló un proyecto de transferencia tecnológica con el apoyo del Ministerio de Salud.

Para el desarrollo del programa de transferencia, se consideró como contenido central el marco de referencia teórico de las consejerías telefónicas y además teorías de aprendizaje para el diseño de las actividades de capacitación. La capacitación se realizó con el apoyo de un módulo de estudio auto dirigido que incluye el marco de referencia teórico de las consejerías telefónicas, y está estructurado en secciones que entregan contenidos, ejercicios y autoevaluaciones ${ }^{(8)}$.

Una parte sustancial de dicho marco de referencia es la Toma de Decisiones en Salud de Ottawa que provee conocimientos específicos respecto a los procesos de toma de decisión y los conflictos decisionales que viven las personas ${ }^{(9)}$. Su propósito es empoderar y apoyar a las personas en el ejercicio de sus derechos en salud y decidir, además de adquirir 0 cambiar, conductas en beneficio de su salud ${ }^{(5)}$.Considera, asimismo, modelos y teorías de cambio de conducta en salud, las que aportan a la comprensión de por qué las personas mantienen o cambian sus conductas en salud, adhieren o no al tratamiento, y cuál es el proceso que viven para realizar estos cambios.

Para apoyar la implementación de la toma de decisiones se trabaja además con la técnica de Entrevista Motivacional, la cual es definida por sus autores como un método directivo, centrado en el cliente, para potenciar y realzar la motivación intrínseca al cambio a través de la exploración y la resolución de la ambivalencia ${ }^{(10)}$. Más que un conjunto de técnicas es un método de comunicación con un enfoque facilitador que evoca el cambio en su forma 
natural. Diversas experiencias señalan que las herramientas motivacionales pueden ser muy efectivas en el apoyo para lograr cambios de estilo de vida de los pacientes ${ }^{(11)}$.

Las tecnologías basadas en este marco de referencia utilizadas en el cuidado de personas con enfermedades crónicas, sumado al uso de tecnologías de atención remota, provee de una oportunidad para entregar una atención centrada en las necesidades del usuario para lograr mejores resultados en salud.

En relación a la intervención telefónica, es importante considerar que al no establecer contacto visual entre profesional y usuario, la identificación de las preocupaciones y los temas relevantes para los usuarios, se basa exclusivamente en la comunicación verbal ${ }^{(12)}$, y por ello, las características de la comunicación verbal del profesional pasan a ser de extraordinaria importancia. Según Katz, así como es complejo el encuentro cara a cara con el usuario, el encuentro telefónico puede serlo aún más, pues hay menos tiempo, no hay posibilidad de lectura del lenguaje corporal y la mayor parte de las veces no hay una oportunidad para segundos pensamientos una vez que el contacto ha terminado y el profesional debe atender la siguiente llamada ${ }^{(13)}$. En este sentido, la capacitación de los profesionales y el entrenamiento de sus habilidades resulta crucial para el resultado clínico de los pacientes.

Desde la perspectiva de las teorías del aprendizaje, el nivel de complejidad de un programa de capacitación para transferencia tecnológica requiere de la incorporación y combinación, en distinta medida y progresivamente, de la Teoría Conductista y de la Teoría Constructivista.

En una primera instancia, se considera la Teoría Conductista, ya que se requerirá observar la conducta del profesional frente a una situación particular, centrando la atención en la percepción y asociación de elementos para el modelaje de la conducta ${ }^{(14)}$.

En una segunda instancia, se requiere que el profesional sea capaz de ir más allá de la asociación de elementos para evaluar su desempeño y el de sus pares, con el propósito de adaptar el modelo propuesto a su realidad local. Para esto, es necesario el enfoque de la Teoría Constructivista ${ }^{(14)}$. Estas acciones orientan al profesional hacia una fase reflexiva donde evaluará su propio aprendizaje y la aplicabilidad de este en la atención directa de usuarios, logrando así la implementación del modelo. Por otra parte, dicha reflexión será fundamental para reestructurar y reacomodar el conocimiento adquirido a la realidad local, adaptándolo a las características propias de su desempeño y la de sus usuarios, sin perder los elementos básicos del modelo ${ }^{(14)}$.

Finalmente, y bajo las perspectivas planteadas, se busca que los profesionales participantes comprendan que el conocimiento adquirido trascienda a una "mera copia de la realidad preexistente" ${ }^{(14)}$, constituyéndose en la base de un proceso interactivo, mediante el cual, podrán interpretar por sí mismos la información adquirida y construir modelos explicativos propios y atingentes a la realidad local ${ }^{(14)}$.

\section{METODOLOGÍA}

Se invitó a profesionales de la salud (enfermeras y nutricionistas) de centros de salud ambulatorios de una comuna de bajos recursos en Chile, a participar en un programa teórico-práctico para incorporar la consejería telefónica de apoyo para personas con condiciones crónicas de salud a su realidad local. El programa consideró las siguientes etapas: a) formación continua para los profesionales en consejería telefónica y el marco 
conceptual que la sustenta, b) entrenamiento de habilidades y c) seguimiento y asistencia específica para la resolución de problemas.

Para ello, se utilizaron diversas metodologías, como: talleres, reuniones, grabación y análisis de consejerías telefónicas con pacientes simulados, acompañamiento de las encargadas del programa durante las consejerías a los pacientes y discusión en pares del desempeño alcanzado ${ }^{(15-16)}$.

La evaluación del programa consideró:

a) Prueba escrita de los contenidos del módulo teórico.

b) Evaluación formativa de la calidad del apoyo decisional entregado por los profesionales, en su intervención telefónica utilizando para ello el instrumento DSAT-cdm (traducido y adaptado de la versión original: "dsatcdm: decision support analysis tool for chronic disease management", stacey, abril 2006, material puesto a disposición por la autora, traducción y adaptación: bustamante, c., 2008, no publicado). EI DSAT-cdm es un instrumento observacional que evalúa el apoyo a la toma de decisiones y las habilidades comunicacionales de los profesionales de la salud en el contexto de intervenciones para personas con condiciones crónicas. las dimensiones evaluadas son: estado y necesidades para la toma de decisiones, necesidades para la implementación y evaluación general de la interacción. Su utilización permite realizar observaciones del progreso en la incorporación de las habilidades. Ppara cada dimensión, el instrumento describe los criterios de valoración que permiten al observador indicar si la dimensión estuvo o no presente durante la interacción entre el profesional y el paciente.

c) Evaluación global del programa desde la perspectiva de los participantes, mediante un cuestionario donde calificaron con nota entre 1 (mínimo) y 7 (máximo) aspectos administrativos, metodológicos y de contenidos del programa.

\section{RESULTADOS}

Entre marzo y agosto del 2009, 14 profesionales de 5 centros de atención primaria realizaron el primer módulo de capacitación teórico práctico intensiva (16 horas). Luego, 10 de ellos continuaron con el seguimiento en terreno para implementar la consejería telefónica. Se realizaron siete sesiones de capacitación práctica y una reunión de evaluación de proceso con el grupo completo de personas que participaron de la capacitación.

Cada profesional capacitado estuvo en promedio 4,5 horas en actividades prácticas en terreno. La programación inicial contemplaba llegar a realizar 10 llamados supervisados con cada uno de ellos, sin embargo, el número de llamados fue menor (entre 1 y 4 por persona), por restricción del tiempo disponible.

Las sesiones de demostración y supervisión de consejerías telefónicas fueron grupales (2 a 3 personas por sesión) y la metodología abarcó: preparación previa al contacto telefónico, ejecución de la consejería y discusión grupal posterior. Esta discusión incluyó el análisis del caso clínico, la evaluación de la consistencia de las intervenciones profesionales durante la llamada con el marco de referencia teórico del modelo y propuestas de posibles intervenciones, en caso necesario. El proceso completo en cada consejería telefónica tomaba entre 45 y 50 minutos, esto explica que durante una sesión de trabajo se alcanzaron a realizar 2 a 3 consejerías como máximo. Para efectos de la capacitación se privilegió la oportunidad de profundizar en la discusión y el análisis, por sobre ejecutar un mayor número de consejerías lo que habría disminuido el tiempo destinado a la actividad grupal. 
Respecto de la evaluación formativa del desempeño, aplicando el DSATcdm, se observó que los participantes obtuvieron un buen logro en los ítemes relacionados con la valoración y/o la entrega de información. Se observó también una evolución desde el énfasis en la valoración de la etapa de la toma de decisión, y las necesidades para la toma de decisiones, especialmente en el área de conocimiento, hacia intervenciones de motivacionales y de apoyo para la toma de decisiones, y diseño de estrategias para la implementación de las decisiones tomadas por los pacientes.

Los participantes del programa evaluaron positivamente ambos módulos de capacitación (teórico y práctico) calificando los aspectos administrativos 6,6, los contenidos con un 6,8 y la metodología con un 6,7 (notas media). Además consideraron que habían alcanzado los objetivos propuestos.

Dado que todos los participantes que realizaron el módulo de seguimiento en terreno presentaron avances cualitativos en su desempeño, se incorporó esta actividad en las prestaciones del centro y se conformaron duplas de trabajo por cada centro, las que definieron los pacientes que serían beneficiarios de la consejería telefónica.

\section{CONCLUSIONES}

La consejería telefónica de apoyo al automanejo para personas con EC, es una tecnología transferible. Esto se puede lograr a través de la aplicación de un programa de capacitación que incluya formación continua, entrenamiento de habilidades y seguimiento de los profesionales. Los resultados positivos de esta capacitación permitieron que cada centro de salud designara tiempo para consejería telefónica a los participantes, asignándole 15 pacientes a cada uno, en el periodo agosto-diciembre 2009.

La transferencia tecnológica supone un desafío para los profesionales de la salud. Considera en una primera instancia lograr la capacidad de los equipos de trasladar lo observado como ideal a la propia realidad, sin perder en el proceso, la esencia de la información a transferir.

La elección de las condiciones apropiadas para lograr dicha transferencia es fundamental para lograr como fin último, que los profesionales sean capaces de ser agentes activos del proceso y no meros observadores.

En este escenario, la utilización de herramientas educativas que incorporen aspectos centrales del proceso de aprendizaje se constituye en elemento esencial para permitir que los profesionales sean capaces de reflexionar e internalizar lo aprendido, construyendo y contribuyendo a un nuevo aprendizaje.

El trabajo en el apoyo a la toma de decisiones en salud y habilidades de entrevista motivacional, canalizado a través de una tecnología de comunicación, favoreció la implementación del modelo de consejería telefónica. Esta transferencia tecnológica es necesaria para que se produzca un cambio sustancial y concreto del modelo de atención habitual hacia una atención integral y centrada en el usuario.

El equipo de trabajo de la Escuela de Enfermería continúa transfiriendo los conocimientos generados en el área de apoyo al automanejo de condiciones crónicas de salud y ampliando el modelo ATAS, con la inclusión del uso de mensajería de texto y el trabajo con personas con pre-diabetes. 


\section{REFERENCIAS}

1. Consejo Internacional de Enfermeras [Internet]. Ginebra: Consejo Internacional de Enfermeras; 2009 [cited 2009 May 12]. Available from: http://www.icn.ch.

2. Oldenburg B, Glanz, K. Diffusion of innovations. San Francisco: John Wiley @ Sons; c2008. Chapter 14, Health behaviour and health education. Theory, research and practice; p. 313-333.

3. Centro REDES [Internet]. Buenos Aires: Centro de estudios sobre ciencia, desarrollo y educación superior; s.f. Medición de actividades de vinculación y transferencia de conocimientos científicos y tecnológicos; 2003 [cited 2009 Jun 29]; [about 18 screens]. Available from: http://www.ricyt.edu.ar/interior/difusion/pubs/elc2003/11.pdf

4. Marshall M. Supporting patients to make the best decisions. BMJ 2011 Apr; 1-2

5. Mendoza S, Jofré V, Valenzuela S. La toma de decisiones en salud y el modelo conceptual de Ottawa. Invest Educ Enferm, 2006 Mar; 24 (1), 86-92.

6. Lange, I. Informe Proyecto MATS FONDEF D04i1194/ 2008. Informe final, Santiago.

7. Lange I, Campos S, Urrutia M, Bustamante C, Alcayaga C, Téllez A, Pérez JC, Villarroel L, Chamorro G, O'Connor A, Piette J. Efecto de un modelo de apoyo telefónico en el auto-manejo y control metabólico de la Diabetes tipo 2, en un Centro de Atención Primaria, Santiago, Chile. Rev. méd. Chile. 2010; 138(6):729-737.

8. Urrutia M, Luarte M, Bustamante C, Borcoski M, Duarte E. Programa de capacitación para profesionales de la salud, Manual de estudio autodirigido. Santiago: Pontificia Universidad Católica de Chile; 2006. 104 p.

9. O Connor AM, Jacobsen MJ. Conflicto decisional: valorando y apoyando a las usuarias que tienen dificultad para tomar decisiones que afectan su salud. Santiago: Pontificia Universidad Católica de Chile; 2004. 41 p.

10. Miller WR, Rollnick S. Motivational interviewing. Preparing people for change. 2nd ed. New York: The Guilford Press; c2002. Chapter 13, Reflections on learning; p. 185-198.

11. Jansink R, Braspenning J, Van der Weijden T, Niessen L, Elwyn G, Grol R. (2009). Nurse-led motivational interviewing to change the lifestyle of patients with type 2 diabetes (MILD-project): protocol for a cluster, randomized, controlled trial on implementing lifestyle recommendations. BCM Health Services Research. 2009 Jan; 9 (19), 1-12.

12. Bottorff JL, Johnson JL, Moffat B, Fofonoff D, Budz, B, Groening M. Synchronizing clinician engagement and client motivation in telephone counseling. Qual Health Res. 2004 Apr; 14 (4), 462-477.

13. Katz H. Telephone medicine. Triage and Training. Philadelphia: F.A Davis Company; c1990. Chapter 1, Overview, Complexity and Opportunity for Quality Improvement; $p$. 3-14.

14. Educar Chile [Internet]. Santiago; Ministerio de Educación de Chile y Fundación Chile; c2001-2012. Teorías del aprendizaje; 2010; s.f [cited 2009 Jun 21]. Available from: http://www.educarchile.cl/web wizzard/ver home.asp?id proyecto=3

15. Salas R, Zulueta P. (1995). La simulación como método de enseñanza y aprendizaje. Rev Cubana Edu Med Sup. 1995 Feb; 9, (1-2).

16. Beullens J, Rethans J, Goedhuys J, Buntinx F. The use of standardized patients in research in general practice. Family Practice. 1997 Feb; 14 (1), 58-62.

ISSN 1695-6141

๑ COPYRIGHT Servicio de Publicaciones - Universidad de Murcia 